\title{
Linfangiectasia Renal Bilateral
}

\section{Bilateral Renal Lymphangiectasia}

\author{
Maira Orozco $^{1}$ M. Victoria Mulqui ${ }^{2}$ Javier De Arteaga ${ }^{3}$ Santiago E. Orozco ${ }^{1}$
}

\author{
${ }^{1}$ Servicio de Diagnóstico por Imágenes, Hospital Privado Universitario \\ de Córdoba, Córdoba, Argentina \\ ${ }^{2}$ Servicio de Clínica Médica, Hospital Privado Universitario de \\ Córdoba, Córdoba, Argentina \\ ${ }^{3}$ Servicio de Nefrología, Hospital Privado Universitario de Córdoba, \\ Córdoba, Argentina
}

Rev Argent Radiol 2020;84:68-70.
Estimados editores,

Reportamos el caso clínico de una paciente de sexo femenino de 47 años, que consultó por dolor lumbar izquierdo progresivo de seis meses de evolución y un episodio de hematuria macroscópica. Tenía antecedentes personales de hipertensión arterial (HTA) de un año de evolución. Al examen físico, presentó dolor a la puño percusión lumbar izquierda. En el laboratorio, hemoglobina y función renal normales, orina con microalbuminuria.

Se le realizó ecografía abdominal (-Fig. 1), que evidenció riñones de tamaño levemente aumentados, con aumento difuso de la ecogenicidad cortical, y en ambos senos renales dilataciones quísticas multitabicadas, de paredes finas, bien definidas. Se decidió complementar estudio con UroTomografía Computada (TC) (-Fig. 2), donde se objetivaron riñones de tamaño levemente aumentado a expensas de numerosos quistes peri-pélvicos bilaterales, de paredes finas, bien definidas, que producen compresión extrínseca del sistema colector, con dilatación proximal secundaria.

Por persistencia de la lumbalgia izquierda, pese al tratamiento analgésico inicial, se le realizó drenaje y alcoholización de los quistes peri-pélvicos renales izquierdos. El líquido claro se analizó, y se obtuvieron 57 células a predominio linfocitario, con cultivos negativos.

La paciente presentó mejoría clínica. En el control a los tres meses con Uro-TC, se evidenció franca reducción volumétrica de los quistes peri-pélvicos izquierdos y menor dilatación del sistema excretor (-Fig. 3).

La linfangiectasia renal, también conocida como linfangiomatosis renal, linfangioma renal, e higroma renal, es un trastorno benigno y poco frecuente. ${ }^{1-3}$ Puede ser congénito o adquirido. ${ }^{1,4}$ Habitualmente es bilateral (90\%) y excepcionalmente está localizado sólo en un sector del
Address for correspondence Maira Orozco, MD, San Lorenzo 135, Córdoba 5000, Argentina (e-mail: mairaorozco16@gmail.com). riñón. Afecta a ambos sexos por igual y puede diagnosticarse a cualquier edad. ${ }^{1,4,5}$ Se han reportado algunos casos de asociación familiar. ${ }^{6,7}$ Poco se conoce sobre su prevalencia, etiopatogenia e historia natural. ${ }^{2}$

Normalmente, el drenaje linfático del riñón, la cápsula renal y los tejidos perirrenales se intercomunican a través de varios troncos linfáticos localizados en el interior del seno renal. Ellos drenan en los ganglios linfáticos paraaórticos, paracavales e interaortocavos. En la linfangiectasia renal, se cree que se presenta una malformación en el desarrollo y alteración en el drenaje de los linfáticos y, en consecuencia, dilatación y ectasia del sistema linfático perirrenal, peripélvico e intrarrenal con la formación de quistes. ${ }^{3,5,7}$

La mayoría de las veces es asintomática. ${ }^{3,4,7}$ Dentro de los síntomas más frecuentes se encuentran: dolor abdominal, hematuria, proteinuria, fiebre, anorexia, pérdida de peso, HTA, masa palpable en flancos, distensión abdominal, ascitis, edema de extremidades inferiores, eritrocitosis y, excepcionalmente, quiluria. ${ }^{4}$ Habitualmente, la función renal es normal, pero hay casos publicados con insuficiencia renal por la compresión del parénquima renal. ${ }^{1,2,4}$

La ultrasonografía evidencia colecciones peripiélicas, perirrenales o intrarrenales uni o multiloculares con septos y ecos en su interior en los casos de detritus y hemorragia. ${ }^{1,5}$ La TC muestra colecciones hipodensas perirrenales y/o en el seno renal, que varían entre 0 y 15 UH (siendo de mayor atenuación en los casos de hemorragia), y sin refuerzo luego de la administración de contraste. Las fases tardías frecuentemente muestran compresión de la pelvis y cálices por los quistes en el seno renal. La corteza renal habitualmente es normal, pero puede estar adelgazada debido a la compresión extrínseca. La resonancia magnética (RM) muestra colecciones hipointensas ponderadas en T1 e received

February 22, 2019

accepted

November 18, 2019
DOI https://doi.org/

10.1055/s-0040-1701273. ISSN 1852-9992.
Copyright (c) 2020, Sociedad Argentina de Radiología. Publicado por Thieme Revinter Publicações Ltda., Rio de Janeiro, Brazil. Todos los derechos reservados.
License terms

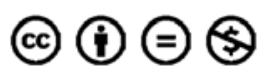




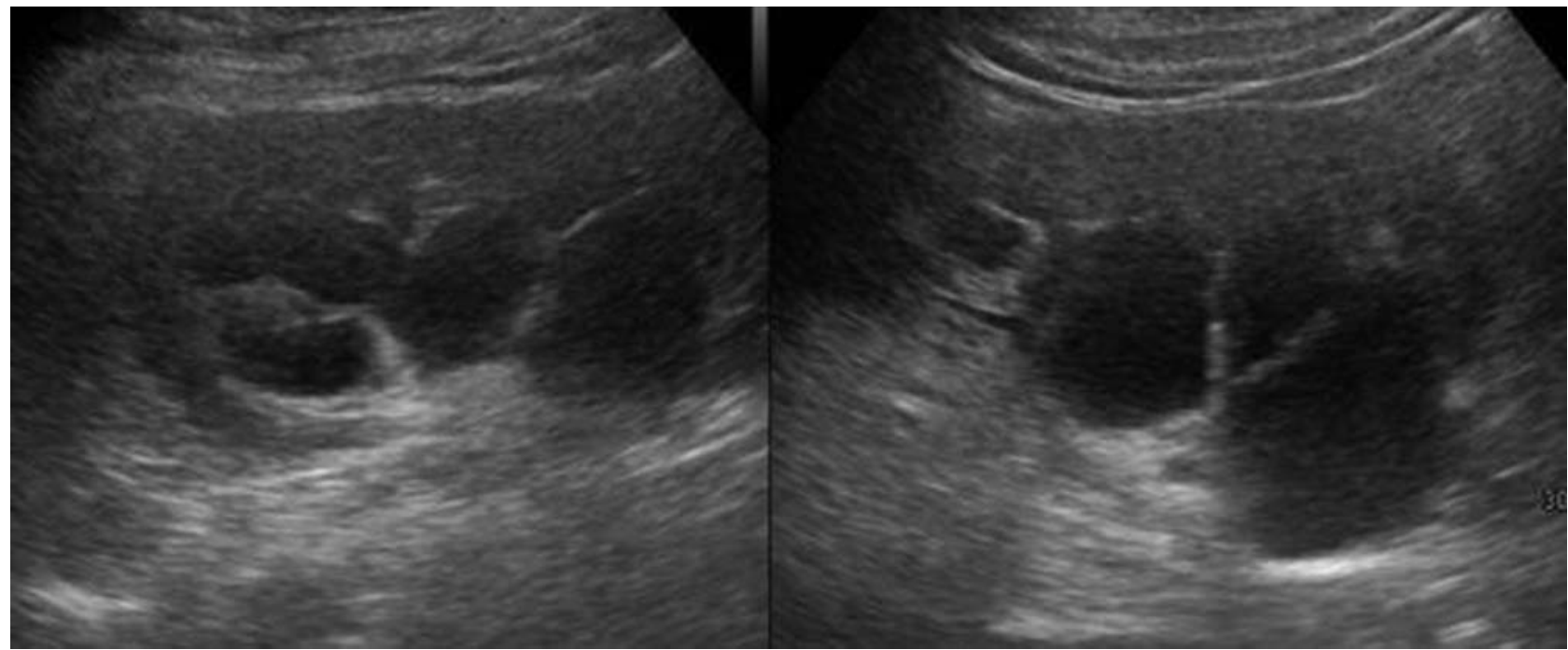

Fig. 1 Ecografía renal. Riñones aumentados de tamaño, presentando a nivel de ambos senos renales múltiples quistes multiloculados, de paredes finas y contenido anecoico homogéneo.

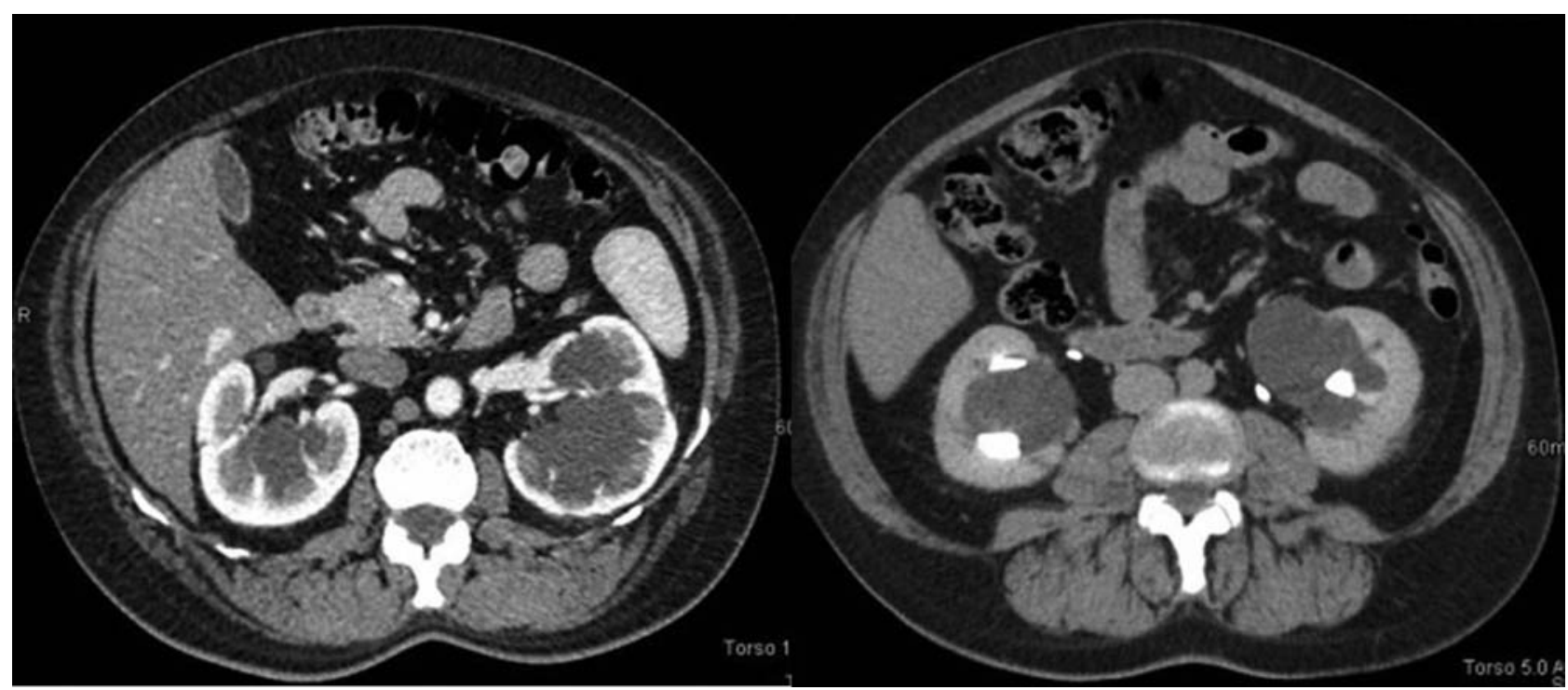

Fig. 2 Uro-TC. Riñones aumentados de tamaño a expensas de numerosos quistes peripiélicos, de paredes finas y contenido homogéneo con densidades de $5 \mathrm{UH}$, que producen compresión con dilatación proximal del sistema excretor.

hiperintensas ponderadas en $\mathrm{T} 2 .^{1,2,4,5}$ La falta de comunicación entre el componente quístico renal y el sistema pielocalicial en la fase excretora de TC o RM con contraste sugieren el diagnóstico de linfangiectasia. ${ }^{4}$

Las complicaciones son raras. Se han descrito hemorragias de los quistes, trombosis de venas renales, HTA dependiente de renina por compresión de las arterias renales (riñón de Page), ascitis, atrofia renal por compresión extrínseca por las masas quísticas y edema de extremidades inferiores por compresión de la vena cava inferior. ${ }^{1-3,5}$

El diagnóstico diferencial es: con la enfermedad renal poliquística, hidronefrosis, quistes simples parapiélicos, enfermedad de von Hippel-Lindau y esclerosis tuberosa, urinoma, linfoma, absceso, lipomatosis del seno renal, hematoma perirrenal, displasia multiquística, nefroma quístico multilocular, nefroblastomatosis y tumor de Wilms. ${ }^{1,2,8}$

La punción y aspiración de los quistes no solo confirma el diagnóstico, sino que también descarta urinoma o absceso. $^{7-9}$ El líquido obtenido es rico en proteínas, tiene pocas células (predominantemente linfocitos), y característicamente presenta altos niveles de renina. Comúnmente no es quiloso, dado que los linfáticos renales no tienen comunicación con los linfáticos mesentéricos. ${ }^{1-3,9}$

Cuando los quistes son sintomáticos, puede efectuarse drenaje percutáneo para disminuir la compresión aunque se reproducen rápidamente. ${ }^{4,5,8,9}$ Puede efectuarse también la "marsupialización" al peritoneo. Intentar resecarlos es dificultoso y las lesiones tienden a sangrar con facilidad, lo 


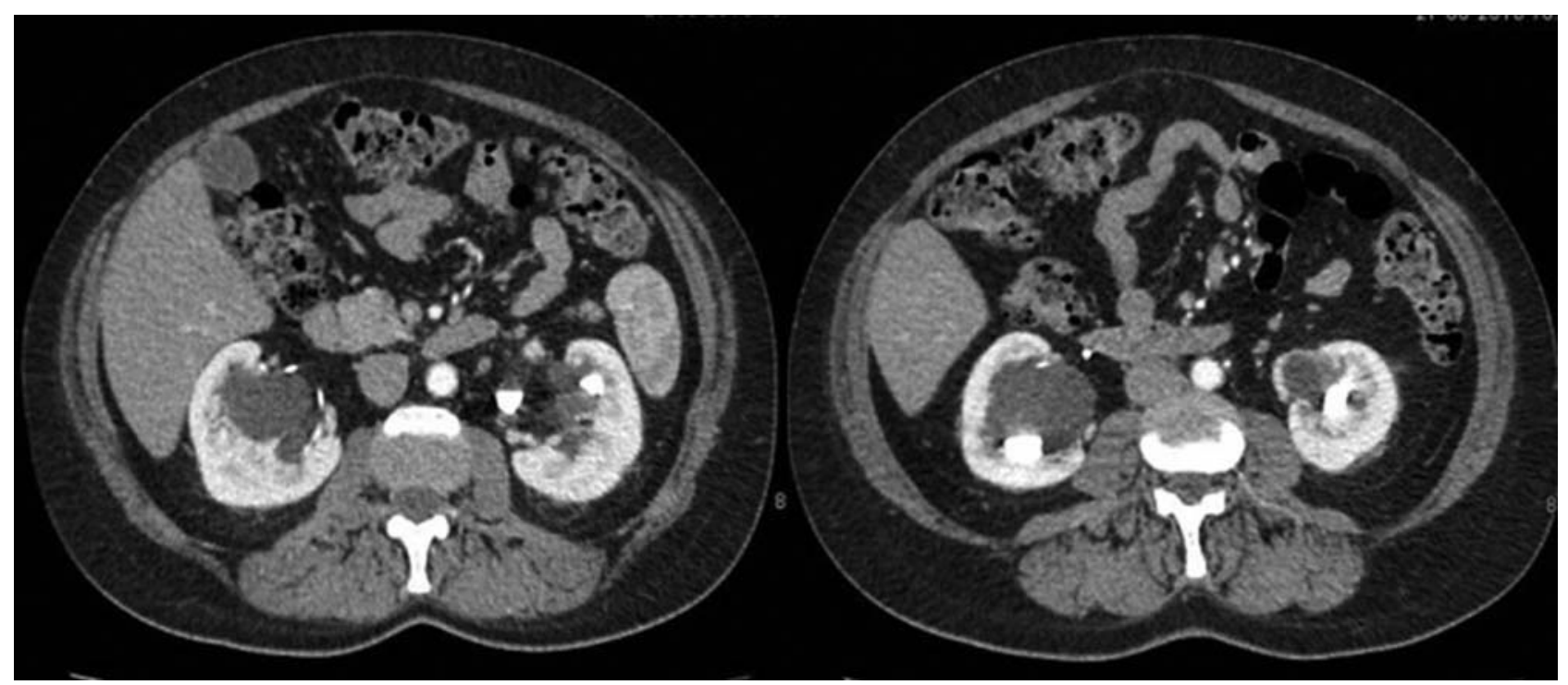

Fig. 3 Control post-punción y alcoholización. Marcada reducción volumétrica de los quistes peripiélicos izquierdos.

que termina obligando a la nefrectomía. ${ }^{1,5,7}$ También se ha comunicado la inyección de sustancias esclerosantes, como alcohol y bleomicina. ${ }^{1}$ La nefrectomía se reserva para casos recurrentes, colecciones complicadas o con difícil manejo de los síntomas. ${ }^{8,9}$

Hay escasa información sobre la evolución a largo plazo. La regresión parcial se ha reportado en casos de neonatos. ${ }^{1,9}$ Se recomienda seguimiento a largo plazo, ya que ocasionalmente la función renal puede deteriorarse. ${ }^{1}$

Confidencialidad de los datos

Los autores declaran que han seguido los protocolos de su centro de trabajo sobre la publicación de datos de pacientes y que todos los pacientes incluidos en el estudio han recibido información suficiente y han dado su consentimiento informado por escrito para participar en dicho estudio.

Conflicto de Intereses

Los autores declaran no tener ningún conflicto de intereses.

\section{Bibliografía}

1 Vega J, Santamarina M. [Unilateral renal limphangiectasia: report of one case]. Rev Med Chil. 2012;140(10):1312-1325

2 Upreti L, Dev A, Kumar Puri S. Imaging in renal lymphangiectasia: report of two cases and review of literature. Clin Radiol. 2008;63 (09):1057-1062

3 Mejia Restrepo J, Lopez Amaya JE, Sepulveda AN, Uribe Velez M, Massaro M. Renal Lymphangiectasia: MDCT and MR Findings. Rev Colomb Radiol. 2011;22(03):1-8

4 Wani NA, Kosar T, Gojwari T, Qureshi UA. Perinephric fluid collections due to renal lymphangiectasia. Am J Kidney Dis. 2011;57(02):347-351

5 Elbanna KY, Almutairi BM, Zidan AT. Bilateral renal lymphangiectasia: radiological findings by ultrasound, computed tomography, and magnetic resonance imaging. J Clin Imaging Sci. 2015;5:6

6 Rastogi R, Rastogi V. Computed tomographic scan in the diagnosis of bilateral renal lymphangiectasia. Saudi J Kidney Dis Transpl. 2008;19(06):976-979

7 Pandya VK, Shah MK, Gandhi SP, Patel HV. Bilateral Renal Lymphangiectasia. J Clin Diagn Res. 2016;10(09):TD01-TD02

8 Jeon TG, Kong H, Park HJ, Kim S, Park WY, Kim SD, et al. Perirenal lymphangiomatosis. World J Mens Health. 2014;32(02):116-119

9 Gorantla R, Yalapati A, Dev B, Joseph S. Case report: Perinephric lymphangiomatosis. Indian J Radiol Imaging. 2010;20(03):224-226 